$$
\begin{array}{r}
\text { Pontifícia Universidade Católica } \\
\text { do Rio de Janeiro }
\end{array}
$$

José Júnior Anastácio Cardoso

"Previsão de estoque inicial de itens eletrônicos sobressalentes reparáveis - Um estudo de caso aplicando o modelo METRIC"

Dissertação de Mestrado

Dissertação apresentada como requisito parcial para obtenção do grau de Mestre (opção profissional) pelo Programa de Pós-Graduação em Engenharia de Produção do Departamento de Engenharia Industrial da PUC-Rio.

Orientador: Prof. Antônio Fernando de Castro Vieira

Rio de Janeiro Setembro de 2009 


$$
\begin{array}{r}
\text { Pontifícia Universidade Católica dio de Janeiro } \\
\text { do Rio }
\end{array}
$$

José Júnior Anastácio Cardoso

\section{"Previsão de estoque inicial de itens eletrônicos sobressalentes reparáveis - Um estudo de caso aplicando o modelo METRIC"}

Dissertação apresentada, como requisito parcial para obtenção do título de Mestre (opção profissional) pelo Programa de Pós-Graduação em Engenharia de Produção da PUC-Rio. Aprovada pela Comissão Examinadora abaixo assinada.

Prof. Antônio Fernando de Castro Vieira Orientador Departamento de Engenharia Industrial - PUC-RIO

Prof. Madiagne Diallo Departamento de Engenharia Industrial - PUC-RIO

Prof. Luiz Felipe Roris Rodriguez Scavarda do Carmo Departamento de Engenharia Industrial - PUC-RIO

Prof. José Eugenio Leal Coordenador Setorial do Centro Técnico Científico - PUC-Rio 
Todos os direitos reservados. É proibida a reprodução total ou parcial do trabalho sem autorização da universidade, do autor e do orientador.

\section{José Júnior Anastácio Cardoso}

Graduou-se em Engenharia Eletrônica e Telecomunicações pelo Instituto Nacional de Telecomunicações de Santa Rita do Sapucaí INATEL - MG em 1983. A partir desta data sua vida profissional foi dedicada às atividades dos setores de treinamento, manutenção e suprimento do Sistema de Controle do Espaço Aéreo Brasileiro, onde participou da concepção e implantação de vários projetos, inclusive do Projeto SIVAM. Atualmente continua dedicado às mesmas atividades no SISCEAB, atuando como membro da organização CTCEA.

Ficha Catalográfica

Cardoso, José Júnior Anastácio

Previsão de estoque inicial de itens eletrônicos sobressalentes reparáveis - Um estudo de caso aplicando o modelo METRIC / José Júnior Anastácio Cardoso; orientador: Antônio Fernando de Castro Vieira. - 2009.

136 f. ; $30 \mathrm{~cm}$

Dissertação (Mestrado em Engenharia Industrial) Pontifícia Universidade Católica do Rio de Janeiro, Rio de Janeiro, 2009.

Inclui bibliografia

1. Engenharia industrial - Teses. 2. Sobressalente. 3. Previsão. 4. Demanda. 5. Poisson. 6. Estoque. 7. Endentamento. 8. Escalão. 9. Manutenção. 10. Modelo probabilístico. 11. Disponibilidade. I. Vieira, Antônio Fernando de Castro. II. Pontifícia Universidade Católica do Rio de Janeiro. Departamento de Engenharia Industrial. III. Título. 
A meus pais,

Meus agradecimentos pela luta e exemplo de dedicação durante a minha formação.

À minha família, Dalva Maria, Germano e Lauro José,

Meus agradecimentos pelo entendimento quanto a minha ausência em vários momentos importantes de suas vidas.

A Deus,

Pela proteção, saúde e misericórdia a mim dispensadas. 


\section{Agradecimentos}

Ao fim de mais um Projeto em minha vida, há muitas pessoas que gostaria de agradecer.

Primeiramente, agradeceria aos dirigentes das entidades CTCEA e CISCEA por terem proporcionado a oportunidade de continuar a minha formação, dando um claro exemplo dos seus comprometimentos com o desenvolvimento das pessoas, fator basilar para o crescimento das partes rumo ao objetivo maior.

Gostaria de agradecer ao Cel $\mathrm{Eng}^{\mathrm{O}}$ Walter Manhães pelo empenho e incentivo para que este projeto fosse levado avante.

Gostaria, também, de agradecer aos colegas, colaboradores internos e externos, Diretores CTCEA e CISCEA pelo apoio e incentivo.

Ao professor Antônio Fernando pelo apoio, orientação, correta postura pedagógica e paciência durante todo o trabalho, colocando-se a todo dispor, inclusive frente às dificuldades do mestrando em alguns momentos em conciliar as obrigações do trabalho e os deveres acadêmicos.

Para evitar cometer esquecimento no agradecimento de algum participante ou colaborador, deixo aqui meus agradecimentos a todos. 


\section{Resumo}

Cardoso, José Júnior Anastácio; Vieira, Antônio Fernando de Castro (Orientador). Previsão de estoque inicial de itens eletrônicos sobressalentes reparáveis - Um estudo de caso aplicando o modelo METRIC. Rio de Janeiro, 2009. 136p. Dissertação de Mestrado (opção profissional) - Departamento de Engenharia Industrial. Pontifícia Universidade Católica do Rio de Janeiro.

A previsão, aquisição e distribuição de sobressalentes de maneira otimizada é primordial para atender eficientemente qualquer sistema logístico de manutenção. Esta dissertação, por intermédio de um estudo de caso, trabalhou dados logísticos reais num espaço amostral extraído de uma proposta técnica e comercial para implantação de uma rede de comunicações em VHF para mostrar a comparação entre o modelo atualmente usado no Sistema de Controle do Espaço Aéreo Brasileiro e o modelo intitulado "MULTI-ECHELON TECHNIQUE FOR RECOVERABLE ITEM CONTROL (METRIC)", originariamente desenvolvido para o cálculo de estoques de sobressalentes para a Força Aérea Americana. A tônica principal desta comparação foi mostrar a diferença existente entre um modelo que trabalha a previsão e distribuição de estoque de itens sobressalentes unicamente enfocando a disponibilidade do item e o outro modelo que enfoca uma análise otimizada de disponibilidade sistêmica. Quando se usa as técnicas da modelagem do METRIC, o produto desta comparação demonstrou fatores de ganho apreciáveis na economia de recursos financeiros, na distribuição estratégica das quantidades de itens sobressalentes nos diversos escalões e na disponibilidade de itens no sistema. Por fim, observando os ganhos nos resultados calculados neste trabalho e considerando a difusão e aceitação do modelo METRIC por várias empresas e organizações governamentais ao redor do mundo, propõe-se que o Sistema Logístico do Controle do Espaço Aéreo passe a considerar o uso do modelo METRIC na implantação de novos sistemas e com isto podendo obter ganhos significativos na área operacional e logística.

\section{Palavras-chave}

Sobressalente, Poisson, estoque, escalão, manutenção, modelo probabilístico, disponibilidade. 


\section{Abstract}

Cardoso, José Júnior Anastácio; Vieira, Antônio Fernando de Castro (Advisor). Initial stock prevision of eletronic repairable spares items A case study with application of the METRIC model. Rio de Janeiro, 2009. 136p. Dissertação de Mestrado (opção profissional) - Departamento de Engenharia Industrial. Pontifícia Universidade Católica do Rio de Janeiro.

The needs, acquisition and distribution of spare parts in an optimized way is very important to support efficiently the logistic maintenance system. This job development, searching a case study that it had its scope in based real data existent on the technical and commercial Proposal for VHF communication Netware, has been worked this subject showing the comparison between the model now used in the Brazilian Air Space Control System and this entitled study "model MULTI-ECHELON TECHNIQUE FOR RECOVERABLE ITEM CONTROL (METRIC)" that it was developed to calculate of spare parts stocks level for the American Air Force. The main core for this comparison was to find the existent difference between the model what predicts the needs and replacement for the available stock levels of the item on hand against the another one that it works with an optimized analysis of the system availability. When it is used the techniques of the METRIC modeling, the product of this comparison demonstrated appreciable improvement of the logistics process and, mainly, for the budget resources, improving the strategic distribution of the spare quantity in the several echelons and in the availability of the items in system. Finally, observing the improvement by the calculated results of this job and considering the diffusion and acceptance of the METRIC model in the several companies and government organizations around the world. At the final, this job proposes that the logistics System of the Air Space Control becomes to consider the using of the tested model in the implementation for the new system and it may obtain significant winnings for the operational and logistics area.

\section{Keywords}

Spares parts, Poisson, Stock control, echelon, maintenance, probabilistics models, availability. 
Pensamentos...

- Todos apregoam que seus produtos são "confiáveis".

- Muitos não quantificam, não testam, não consideram as normas pertinentes, e não julgam a Confiabilidade, a Mantenabilidade e a Disponibilidade de seus produtos e serviços.

- Poucos consideram a influência dos sensores e das Redundâncias ativas ou passivas. As garantias geralmente cobrem apenas falhas na "infância" dos produtos, e não os serviços a serem prestados na sua vida útil.

- Algumas assistências técnicas se preocupam mais com o faturamento de sobressalentes, na capacidade limite de insatisfação dos clientes.

- Poucas empresas se preocupam em adotar um treinamento eficaz para a análise de falhas e reparos (manutenção), normalmente gerando grande quantidade de relatórios inconsistentes e graves conflitos entre setores e pessoas.

A abordagem da nova qualidade do produto deve ser corajosa, muitas coisas inovadoras devem ser realizadas... o mercado mudou, os consumidores saberão selecionar os competentes e honestos. 


\section{Sumário}

1. Introdução 13

1.1. Introdução Geral ao Tema 13

$\begin{array}{ll}\text { 1.2. Objetivo Geral } & 18\end{array}$

1.3. Objetivos específicos 18

$\begin{array}{ll}\text { 1.4. Importância do Trabalho } & 18\end{array}$

$\begin{array}{ll}\text { 1.5. Estrutura da dissertação } & 20\end{array}$

2. Situação Atual 21

2.1. Estrutura logística no SISCEAB 21

2.1.1. DECEA 23

2.1.2. PAME 23

2.1.3. Regionais 25

2.1.4. Sítios 26

2.1.5. Depósito da Aeronáutica 26

2.1.6. CABW e CABE 27

2.1.7. CISCEA 27

2.2. Atividades de Manutenção no SISCEAB $\quad 27$

2.3. Sistema de Controle Logístico do SISCEAB 28

2.4. Catalogação 29

2.5. Definição e Quantificação de uma Lista Inicial de Sobressalentes 30

2.5.1. Metodologia de Previsão utilizada pela CISCEA 31

2.5.2. Cálculo da quantidade demandada de sobressalente 35

2.5.3. Estudo de caso

3. Proposta de Mudança 51

3.1. Conceitos básicos para Modelagem do "METRIC"

3.1.1. Estrutura e definição de variáveis para o modelo "METRIC" 53

3.1.2. Demanda 53

3.1.3. Política de Manutenção, Tempo de Reparo e Atividades de
Manutenção

3.1.4. Média e Variância $\quad 56$

3.1.5. Distribuição de Poisson 56

3.1.6. Teorema de PALM

3.1.7. Nível de estoque $\quad 58$

3.1.8. Medidas de desempenho para Item e Sistema $\quad 59$

3.2. Modelos para Definição de Estoques de Itens Reparáveis 61

3.2.1. Modelo de Estoque Base $\quad 61$

3.2.2. METRIC $\quad 64$

3.3. Exemplo de aplicação do METRIC $\quad 72$

3.4. VARI-METRIC $\quad 86$

3.4.1. Teoria para Múltiplos Escalões $\quad 87$

3.4.2. Procedimentos e Definições para uso do VARI-METRIC 90

3.4.3. Disponibilidade $\left(\mathrm{A}_{0}\right) \quad 97$

3.4.4. Otimização 98

3.5. Solução do Caso Sistema Rádio VHF pelo Modelo VARI-METRIC 98 
3.5.1. Preparação dos dados para VARI-METRIC

4. Conclusão e Sugestões

5. Referências bibliográficas

Anexo I

Memória de Cálculo para Modelo CISCEA 


\section{Lista de figuras}

Figura 2.1 - Diagrama de Estrutura do SISCEAB 24

Figura 2.2 - Estrutura de Manutenção do Sistema Rádio 44

Figura 3.1 - Cenário do Modelo de Estoque Base 62

Figura 3.2 - Cenário do "METRIC" 66

Figura 3.3 - Gráfico do Sistema por Nível de Orçamento 85

Figura 3.4 - Sentido de cálculo das demandas e faltas 93

Figura 3.5 - "Fill Rate" 113

Figura 3.6 - Saídas para o Objetivo $3 \quad 119$

Figura 3.7 - Saídas para o Objetivo 4 


\section{Lista de tabelas}

Tabela 2.1- Critérios da CISCEA para distribuição de sobressalentes 34

Tabela $2.2-P_{s}(x)$ sugerida em função de $A_{0} \quad 38$

Tabela 2.3 - Critérios para dimensionamento dos Itens Sobressalentes 43

Tabela 2.4 - MTBF para os módulos dos Rádios 47

Tabela 2.5 - Quantidade de Sobressalentes por Localidade $\quad 49$

Tabela 3.1 - Dados coletados sobre Faltas $\quad 60$

Tabela 3.2 - Dados para o Problema - METRIC $\quad 73$

Tabela 3.3 - Resumo da Quantidade e Distribuição de Estoque 85

Tabela 3.4 - Estruturação e dados sobre os equipamentos 101

Tabela 3.5 - Escalonamento e Tipo de Estação 102

Tabela 3.6 - Escalonamento e Tempos de Serviços 103

Tabela 3.7 - Valores para "MRR6" 105

Tabela 3.8 - Valores dos "NRTS" 106

Tabela 3.9 - Valores do "Repair Cycle Time - RCT" 106

Tabela 3.10 - Valores dos "Order \& Ship Time" 107

Tabela 3.11 - Condições objetivo para o Projeto 108

$\begin{array}{ll}\text { Tabela } 3.12 \text { - Resumo das saídas para o Projeto } & 108\end{array}$

Tabela 3.13 - Resumo das saídas do Projeto para "Fill Rate" de 90\% 109

$\begin{array}{ll}\text { Tabela } 3.14 \text { - Sobressalentes por Sítio } & 110\end{array}$

Tabela 3.15 - Saídas por Interação $\quad 111$

Tabela 3.16 - Resumo das saídas para o Projeto 114

Tabela 3.17 - Resumo das Saídas do Projeto para Disponibilidade $\begin{array}{ll}\text { Operacional de 90\% } & 114\end{array}$

Tabela 3.18 - Resumo das Saídas para o Projeto 115

Tabela 3.19 - Resumo das saídas do Projeto para A $-90 \%$ e “Avg. Delay” 116

-15 dias

$\begin{array}{ll}\text { Tabela } 3.20 \text { - Saídas para Interação } & 117\end{array}$

$\begin{array}{ll}\text { Tabela } 3.21 \text { - Resumo das saídas para o Projeto } & 120\end{array}$

Tabela 3.22 - Saídas por Interação 120

Tabela 3.23 - Sobressalentes por sítio 122 